\title{
Riskier Than We Think? The Relationship Between Risk Statement Completeness and Perceptions of Direct to Consumer Advertised Prescription Drugs
}

\author{
JOEL J. DAVIS
}

School of Communication

San Diego State University

San Diego, California, USA

\begin{abstract}
Direct to consumer (DTC) prescription drug advertising is one of the fastest growing categories of advertising. Expenditures have increased from about $\$ 25$ million in 1992 to nearly \$2 billion in 1999. Given strong evidence of consumerdriven demand for advertised prescription drugs, research was conducted to assess the extent to which DTC prescription drug advertising provides consumers with the information they need to make an informed evaluation of an advertised drug's relative benefits and risks.

Two studies explored the relationship between the completeness of the statement describing drug-associated side effects (the "risk statement") and consumers' perceptions of a drug's safety and appeal. The research manipulated risk statement completeness with regard to the incidence levels of side effects mentioned in the statement (which in turn affected the number of side effects mentioned) and the presence or absence of a numeric indicator of side effect incidence. The research strongly suggests a direct relationship between risk statement completeness and consumers' perceptions of drug safety and appeal. Consumers rate the safety and appeal of drugs described with an incomplete risk statement significantly more positively than comparable drugs described with a more complete risk statement. Implications of the research for the regulation and presentation of DTC prescription drug advertising and advertiser communication practices are discussed.
\end{abstract}

One of the fastest growing forms of consumer advertising is DTC prescription drug advertising - a pharmaceutical company's presentation of prescription drug information to the general public through consumer-oriented media (Pierpaoli, 1986). Media expenditures for DTC prescription drug advertising have increased nearly twenty-fold during the 1990s, growing from about \$25 million in 1992 to almost \$1.9 billion dollars in 1999 (Okie, 2000). Moreover, advertising expenditures are expected to continue to increase because of recent Food and Drug Administration (FDA) regulatory changes. The FDA has finalized guidelines for radio and television prescription drug advertising, which allows advertisers to describe their drug's benefits without providing long, detailed descriptions of the drug's side effects (FDA, 1999).

Sales of advertised prescription drugs often increase dramatically after the introduction of DTC advertising (Liebman, 2000). Claritin, for example, was the leading prescription drug advertiser in the first half of 1998 and, in that time period, its sales rose $32 \%$. Allegra and Zyrtec, also heavy advertisers, saw sales rise $100 \%$ and $56 \%$,

Address correspondence to Joel J. Davis, School of Communication, San Diego State University, San Diego, CA 92182-4561, USA. E-mail: jdavis@mail.sdsu.edu 
respectively (Freudenheim, 1998). Importantly, in spite of the fact that an advertised drug must be prescribed by a physician, evidence suggests that the rise in sales of DTC advertised prescription drugs is consumer driven. Five related trends help to explain how DTC advertising affects product sales:

- High levels of advertising increase consumers' awareness of prescription drugs and the conditions that they treat. A recent survey conducted by the National Consumer's League found that nearly $80 \%$ of consumers say that they are aware of prescription drug advertising (Brody, 1998) and the average consumer recalled seeing ads for five different prescription drugs (Center for Drug Evaluation and Research, 1999).

- Awareness leads consumers to talk with their doctors about advertised conditions. Patient visits have increased most rapidly in areas related to the conditions treated by the most heavily advertised DTC drugs. In the seven most heavily advertised groups of DTC products (cholesterol, smoking cessation, osteoporosis, hair loss, ulcers, postmenopausal problems, and depression) patient visits increased $22 \%$ versus a $2 \%$ overall patient increase when compared with the prior year (Freudenheim, 1998; Holmer, 1999).

- During physician visits, consumers take the initiative in requesting the advertised drug. High levels of drug advertising awareness is associated with a greater likelihood of requesting a specific drug (Peyrot, Alperstein, Van Doren, \& Poli, 1998). Eighty percent of adults say they would be likely to talk with their doctor if they saw an advertisement for a drug that treated a condition that was bothering them (CDER, 1999). This predisposition may explain the results of the IMS Health survey of 2,500 physicians, which found that more than half of all physicians $(53 \%)$ reported a "significant increase" in patients requesting drugs by brand name. Importantly, this increase appears to be related to the impact of DTC advertising.

- Consumers are more likely to request highly advertised drugs. Survey research has linked advertising expenditures and drug requests. Two of consumers' most requested drugs (Claritin and Allegra) are also the most heavily advertised (Hollon, 1999). Moreover, the 10 most heavily advertised drugs (in 1998) account for $22 \%$ of the total increase in spending by consumers for all drugs between 1993 and 1998 (Zendle, 1999).

- Consumers don't give up. Most consumers are successful in obtaining the requested drug. Survey data estimate that between half and three-quarters of all requests are approved by a physician (Brody, 1998, "National Survey," 1998). Moreover, there are indications that consumers who are unable to obtain an advertised drug from their own physician meet the denial with "suspicion and hostility" (Neergaard, 1999) and "shop around" until they find a physician willing to prescribe the desired drug (Cohen, 1990; Sheffet \& Reece, 1994).

Marketplace data show that DTC prescription drug advertising has affected how consumers perceive, request, and obtain prescription drugs. However, to what extent does this advertising allow consumers to evaluate accurately the risks and benefits associated with alternative, competing drugs and to develop appropriate brand requests and preferences? This question is important, as research has shown that consumers are more likely to make unsound medical decisions when they have incomplete or imperfect knowledge of the risks and benefits associated with alternative options (Viscusi, Magat, \& Huber, 1986). We examine one advertiser practicethe completeness or lack of completeness of the risk information presented in DTC 
prescription drug advertising - and the effect of this practice on consumers' perceptions of drug safety and drug preference. The exploration of this issue extends prior research on consumers' awareness of and reactions to DTC prescription drug advertising (see, for example, Alperstein \& Peyrot, 1993; Everett, 1991; Perri \& Dickson, 1988; Perri \& Nelson, 1987) and is conducted within the context of the FDA's approach to the regulation and evaluation of DTC drug advertising.

\section{DTC Prescription Drug Advertising Regulation and Risk Statement Completenes}

The content of DTC prescription drug advertisements is governed by regulations issued by the FDA. The FDA examines prescription drug advertising to make certain that from a content perspective the advertisement is not deceptive, misleading, or does not in other ways violate any applicable rules and regulations. FDA regulations list 33 ways in which any prescription drug advertisement may be false or misleading (Code of Federal Regulations, 1997). Beyond these criteria that apply to all DTC drug advertisements, product-specific print advertisements, that is, advertisements that mention the drug's name and present information on the drug's potential benefits, safety, side effects, and risk, are considered deceptive or misleading if they fail to present fair balance within the text of the advertisement.

Fair balance is one of the most important aspects of the FDA's prescription drug advertising regulations. Here, the FDA examines an advertisement to determine if it presents an unbiased and balanced account of the risks and benefits associated with drug usage. From the perspective of the FDA, a product-specific advertisement

fails to present a fair balance between information relating to side effects and contraindications and information relating to effectiveness of the drug in that the information relating to effectiveness is presented in greater scope, depth or detail ... and this information is not fairly balanced by a presentation of a summary of true information relating to side effects and contraindications of the drug. (Code of Federal Regulations, 2000, p. 76)

Fair balance, therefore, requires that a DTC prescription drug print advertisement present a balance of risk and benefit information within the context of the advertisement. Risk information typically is presented in a risk statement, a one or two sentence presentation of the side effects associated with drug usage. The FDA's regulations governing prescription drug advertising do not specify the specific form or content of the risk statement and, as a result, advertisers have considerable latitude in the form and content of the side effects reported in a DTC drug advertisement's risk statement. Advertisers must present a balance of drug benefits and risks (side effects). They are, however, under no compulsion to report all of side effects or to even report all major side effects.

What then is the relationship between fair balance and completeness in the reporting of side effects? From a content perspective, completeness in side effect reporting is not a specified and explicit component of the FDA's fair balance criterion. Advertisers can satisfy the FDA's fair balance criterion and still present incomplete risk information, for example, by omitting a relatively high incidence side effect or failing to present a fair and representative listing of all side effects. But does the presentation of side effects in this manner really achieve "fair balance?" We argue that (1) fair balance is only achieved when a consumer, upon reading or seeing 
a DTC prescription drug advertisement, clearly understands his or her likelihood of suffering drug-related side effects and is able to place the risk of these side effects in the context of anticipated drug-related benefits and that (2) this understanding is achievable only when a risk statement presents a complete description of the risks associated with a particular drug's usage.

The argument that fair balance requires a complete (or at least more complete) disclosure of risk information is predicated on the assumption that consumer attitudes toward an advertised drug are affected by the completeness of the risk statement. If attitudes toward drug safety, benefits, and appeal are affected by risk disclosure completeness, then an argument can be made that fair balance is not achieved in the context of incomplete risk statements. On the other hand, if consumer attitudes are not affected by the characteristics of the risk statement, that is, if attitudes toward the advertised drug are the same when confronted with complete and incomplete risk statements, then the issue of completeness becomes less significant.

There is no commonly accepted definition of "completeness" with regard to DTC prescription drug advertising risk statements nor is there a consensus as to a methodological approach for exploring the effects of completeness on consumers' attitudes toward the advertised drug. As a result, this paper presents the results of two empirical studies that explore the relationship of risk statement completeness and subsequent attitudes toward an advertised drug. Each study provides a different operational definition of "complete risk statement" and utilizes a different methodological approach. Both studies seek to answer the question: "To what extent does the completeness of side effect reporting affect consumer perceptions of drug safety and drug preference?

\section{Methods: Study One}

Study One examined the effect of specifying a minimal reporting level for drugrelated side effects on consumers' preference for an advertised prescription drug. The specification of this level, in this study, was identified through qualitative research. Individual interviews were conducted with 37 undergraduate and graduate students at a major western university. These students (who ranged in age from 22 to 43) indicated that an incidence level of $3 \%$ was considered their baseline of concern. Most said that they were concerned and wanted to know about drug-related side effects occuring at a rate of $3 \%$ or greater; most were not concerned about side effects occurring at less than a $3 \%$ level of incidence. As a result, a complete risk statement was defined operationally in this study as "a statement that presented all drug-related side effects occurring at a rate of $3 \%$ or greater." Finally, in terms of setting a minimal level of side-effect concern, it should be noted that although the generalizability of these students' responses is limited given their nonrandorn selection, the consistency of their responses nevertheless provides sufficient direction for the setting of a minimal level of concern with regard to side effect incidence.

\section{Creation of Complete and Incomplete Risk Statements}

Reflecting the study's operational definition of "risk statement completeness," two different risk statements were developed for each of eight drugs that, at the time of the research, were appearing in DTC prescription drug advertisements.

The first risk statement (for analytical purposes labeled the "incomplete risk statement") was taken directly from the drug's DTC advertisement and as a result 
reflected current advertiser practice. Incomplete risk statements always presented a subset of all potential side effects, and in all cases, not all side effects at or above the $3 \%$ criterion were presented. A typical incomplete risk statement was, "Like any prescription drug, Fosamax may cause side effects. The most common side effects are: stomach and muscle, bone or joint pain."

The second risk statement for each drug (for analytical purposes labeled the "complete risk statement") was identical to the first except that all side effects occurring at an incidence of $3 \%$ or more were included in the description. These side effects were identified from data provided in the brief summary that accompanied each drug's DTC advertisement. The complete risk statement for Fosamax was

TABLE 1 Product Descriptions and Risk Statements Used in Study One

\section{Claritan-D}

Description

Incomplete Risk Statement

Complete Risk Statement

\section{Cadura}

Description

Incomplete Risk Statement

Complete Risk Statement
Claritan-D is a prescription drug that helps with seasonal allergies. It takes care of all your symptoms: your stuffy, congested nose . . those itchy, watery eyes . . . that runny nose . . . your itchy ears/palate . . . and all that sneezing. Claritan-D contains pseudoephedrine sulfate. Too much pseudoephedrine sulfate can cause nervousness, sleeplessness, and dizziness. Claritan-D contains pseudoephedrine sulfate. Too much pseudoephedrine sulfate can cause nervousness, sleeplessness, dizziness, dry mouth pharyngitis, coughing, fatigue, nausea, anorexia, and dysmenorrhea.

Cadura is a prescription drug product that helps treat frequent nighttime urination due to $\mathrm{BPH}$ (Benign prostatic hyperplasia). Cadura is a simple, once-daily tablet that quickly relieves urination problems caused by BPH, meaning your bladder can empty completely and you won't have to wake up as often at night to go to the bathroom. Cadura can cause a sudden drop in blood pressure at the beginning of treatment. You may feel dizzy, faint or light-headed, particularly after getting up from a chair or bed.

Cadura can cause a sudden drop in blood pressure at the beginning of treatment. You may feel dizzy, faint or light-headed, particularly after getting up from a chair or bed. Other side effects you could have while taking Cadura include: fatigue (tiredness), swelling of the feet and shortness of breath. You should not drive or do any hazardous tasks until you are used to the side effects of the medication. 
TABLE 1 Continued

\section{MetroGel}

Description

Incomplete Risk Statement

Complete Risk Statement

Fosamax

Description

Incomplete Risk Statement

Complete Risk Statement
MetroGel is a prescription drug product that helps treat roseacea-a skin condition that causes broken blood vessels, acne-like bumps, and redness in the middle areas of the face. Roseacea symptoms may come and go, but without treatment may get worse over time.

Patients using MetroGel experienced the following individual side effects: skin discomfort (burning and stinging), redness, skin irritation, irritation or worsening of roseacea.

Patients using MetroGel experienced the following individual side effects: skin discomfort (burning and stinging), redness, skin irritation, irritation or worsening of roseacea. In addition, the following adverse experiences have been associated with the used of metronidazole: burning; dryness; transient redness, taste, tingling, or numbness of extremities; and nausea.

Fosamax is a prescription drug product that helps treat osteoporosis. Osteoporosis is caused when women lose bone mass faster than it is replaced. During the first five years after menopause, some women may lose as much as $15 \%$ of their bone density. Fosamax is a treatment that's nonhormonal and proven to restore lost bone in many women past menopause.

Like any prescription drug, Fosamax may cause side effects. The most common side effects are: stomach and muscle, bone, or joint pain. Like any prescription drug, Fosamax may cause side effects. The most common side effects are: stomach and muscle, bone, or joint pain. Other, less frequently reported side effects are nausea, heartburn, irritation or pain of the esophagus (the tube that connects your mouth to your stomach), vomiting, difficulty swallowing, a full or bloated feeling in the stomach, constipation, diarrhea, and gas. 
TABLE 1 Continued

\section{Imitrex}

Description

Incomplete Risk Statement

Complete Risk Statement

\section{Relafen}

Description

Incomplete Risk Statement

Complete Risk Statement

\section{Sporanox}

Description

Incomplete Risk Statement

Complete Risk Statement
Imitrex is a prescription drug product that helps relieve migraine attacks. Imitrex tablets not only relieve the pain, but also the nausea and sensitivity to light that often accompany migraine. The most frequently seen side effects are tingling and nasal discomfort.

The most frequently seen side effects are tingling and nasal discomfort. Other side effects include feelings of pressure, heaviness and tightness, warm/hot sensations, cardiovascular palpitations and flushing, eye irritation, and visual disturbance.

Relafen is a prescription drug product that helps treat arthritis pain. Relafen helps relieve the pain in joints - hands, feet, hips, and knees-wherever you need it. In clinical trials, it reduced pain and inflammation for thousands of arthritis suffers around the world.

While most people tolerate Relafen well, some may experience side effects such as diarrhea, heartburn and abdominal pain. In some cases there could be more serious side effects such as stomach ulcers and intestinal bleeding.

While most people tolerate Relafen well, some may experience side effects such as diarrhea, dyspepsia, abdominal pain, constipation,

flatulence, nausea, positive stool guiaic, dizziness, headache, puritus, rash, tinnitus, and edema. In some cases there could be more serious side effects such as stomach ulcers and intestinal bleeding.

Sporanox is a prescription drug product that helps get rid of toenail fungus. Sporanox has been proven successful in kicking nail fungus - thick, yellowing brittle nails.

In clinical trails involving patients with thick, hard, yellowish and/or brittle nails, the following adverse effects led to either a temporary or permanent discontinuation of treatment: elevated liver enzymes, gastrointestinal disorders, and rash. In clinical trials involving patients with thick, hard, yellowish and/or brittle nails, the following adverse effects led to either a temporary or permanent discontinuation of treatment: nausea, vomiting, diarrhea, edema, fatigue, headache, elevated liver enzymes, and rash. 
TABLE 1 Continued

\section{Atrovent Nasal Spray}

Description

Incomplete Risk Statement

Complete Risk Statement
Atrovent Nasal Spray is a prescription drug product that treats runny noses. Atrovent Nasal Spray's special inhibitors provide effective relief without insomnia or drowsiness.

Though most Atrovent Nasal Spray users experience trouble-free relief, nasal dryness and mild nose bleeds are potential side effects. In some cases there could be more serious side effects such as stomach ulcers and intestinal bleeding.

While most people tolerate Atrovent well, some may experience side effects such as diarrhea, dyspepsia, abdominal pain, constipation, flatulence, nausea, positive stool guiaic, dizziness, headache, puritus, rash, tinnitus, and edema. In some cases there could be more serious side effects such as stomach ulcers and intestinal bleeding.

Like any prescription drug, Fosamax may cause side effects. The most common side effects are: stomach and muscle, bone, or joint pain. Other, less frequently reported side effects are nausea, heartburn, irritation or pain of the esophagus (the tube that connects your mouth to your stomach), vomiting, difficulty swallowing, a full or bloated feeling in the stomach, constipation, diarrhea and gas.

Each incomplete and complete risk statement was preceded by an identical product description. This description named the drug and provided a brief overview of the condition treated by the drug and the drug's major benefit(s). The product description for Fosamax was

Fosamax is a prescription drug that helps treat osteoporosis. Osteoporosis is caused when women lose bone mass faster than it is replaced. During the five years after menopause, some women may lose at much as $25 \%$ of their bone density. Fosamax is a treatment that's non-hormonal and proven to restore lost bone in many women past menopause.

The product descriptions and risk statements for each of the eight drugs used in Study One are shown in Table 1.

\section{Questionnaire}

It was believed that one potential source of bias might be related to the overall length of the risk statement. Since complete risk statements were always longer than incomplete risk statements, the mixing of incomplete and complete risk statements in the same questionnaire potentially could draw attention to the shorter, incomplete descriptions. As a result, all complete risk statements appeared on one questionnaire and all incomplete risk statements appeared on a second questionnaire. The order of drug presentation within the questionnaires was determined by a 
random drawing; the final order of presentation was identical for both forms of the questionnaire.

\section{Measure}

Each product description (on both questionnaires) ended with the following question: "If you or someone you know had [name of medical problem from product description inserted here] how likely or unlikely would you be to recommend or purchase [name of drug inserted here]?"

This question was followed by a seven-point Likert scale with endpoints of "Extremely likely to recommend or purchase [name of drug]" and "Extremely unlikely to recommend or purchase [name of drug]." Several aspects of this measure merit noting.

- The use of "recommend or purchase" increased the relevance of the question to each respondent. Although some members of the sample were unlikely to exhibit a particular described medical condition, all members of the sample conceivably could be in the position of knowing someone with the condition, and thus might be in the position of recommending the advertised drug.

- "Recommend or purchase" goes to the core of the Federal Trade Commission's (FTC's) approach to advertising regulation, the measurement of materiality, which is the "extent to which a representation, omission or practice influences consumer behavior or purchasing patterns" (FTC, 1983).

- "Recommend or purchase" provides an easy-to-evaluate summary measure of drug perceptions.

\section{Procedure}

Respondents completed each questionnaire individually. Recruited respondents were taken to a small interview room and were asked to read the questionnaire directions and complete the questionnaire. The questionnaire directions read, "Please read each question and answer as instructed. There is no right or wrong answer to each question." Respondents were given an unlimited amount of time to complete the questionnaire, which they gave to a trained interviewer upon completion. Questionnaires were checked for completeness and problem questionnaires (three in this study because of missing data) were returned to the respondent for revision.

\section{Results}

Demographic Characteristics. The sample for Study One consists of 140 adults recruited via mail intercept. No incentive was offered for study participation. Seventy-five adults responded to the questionnaire containing incomplete risk statements, and 65 responded to the questionnaire that contained the complete risk statements. Importantly, there were no significant differences in demographics between the two versions of the questionnaire (see Table 2). The samples for both questionnaires consisted of approximately equal numbers of men and women with a broad range of education, age, and income. The results of chi-square tests comparing the demographics of the two questionnaires were as follows: gender, chi square $=1.8, p=$ n.s., $d f=1$; education, chi square $=4.3, p=$ n.s., $d f=5$; income, chi square $=9.4, p=$ n.s., $d f=6$. A $t$ test for age was also not significant $(t=1.85$, 
TABLE 2 Demographic Characteristics of Respondents: Studies One and Two

\begin{tabular}{|c|c|c|c|}
\hline & \multicolumn{2}{|c|}{ Study One } & \multirow[b]{2}{*}{ Study Two } \\
\hline & Incomplete & Complete & \\
\hline & $\begin{array}{l}\text { Description } \\
(n=75) \\
(\%)\end{array}$ & $\begin{array}{c}\text { Description } \\
(n=65) \\
(\%)\end{array}$ & $\begin{array}{c}(n=58) \\
(\%)\end{array}$ \\
\hline \multicolumn{4}{|l|}{ Gender } \\
\hline Female & 44 & 45 & 47 \\
\hline Male & 56 & 55 & 53 \\
\hline \multicolumn{4}{|l|}{ Age } \\
\hline 18 to 24 & 31 & 23 & 24 \\
\hline 25 to 34 & 17 & 25 & 34 \\
\hline 35 to 44 & 16 & 15 & 14 \\
\hline 45 to 54 & 7 & 6 & 7 \\
\hline 55 and older & 29 & 31 & 21 \\
\hline \multicolumn{4}{|l|}{ Education } \\
\hline High school or less & 33 & 37 & 59 \\
\hline Some college & 45 & 34 & 15 \\
\hline Graduated college & 22 & 29 & 26 \\
\hline \multicolumn{4}{|l|}{ Income } \\
\hline Less than $\$ 12,000$ & 18 & 20 & 25 \\
\hline$\$ 12,000$ to $\$ 24,999$ & 42 & 32 & 33 \\
\hline$\$ 25,000$ to $\$ 39,999$ & 21 & 24 & 15 \\
\hline$\$ 40,000$ to $\$ 59,999$ & 17 & 12 & 21 \\
\hline$\$ 60,000$ and over & 2 & 7 & 6 \\
\hline Refused & 0 & 5 & 0 \\
\hline
\end{tabular}

$p=$ n.s., $d f=138$ ). The comparability of the samples for the two questionnaires is important because it eliminates demographic variations across questionnaires as a potential explanation for any differences seen in responses to the incomplete risk statements (shown on one questionnaire) and complete risk statements (shown on the second questionnaire).

Effect of Risk Statement Completeness on Intent to Recommend or Purchase. Risk statement completeness significantly affected intent to recommend or purchase (Table 3). In seven of the eight cases, consumers were significantly more likely to "recommend or purchase" a drug when the drug's description was accompanied by an incomplete versus complete risk statement (all $p$ values were .05 or less). The eighth case showed no difference between the two descriptions $(p=.11)$ although the direction of the mean scores was consistent with the prior seven cases; that is, consumers were more likely to "recommend or purchase" the drug when the drug's description was accompanied by an incomplete versus complete risk statement.

Risk statement completeness, however, did not affect directionality of response as all mean ratings were greater than the neutral midpoint of the scale; that is, all mean ratings indicated a positive likelihood to recommend or purchase. Thus, 
TABLE 3 Study One: "Intent to Recommend or Purchase" as a Function of Side Effect Description Completeness

\begin{tabular}{lcccc}
\hline & \multicolumn{3}{c}{ Mean Rating $^{a}$} & \\
\cline { 2 - 3 } \multicolumn{1}{c}{ Drug } & $\begin{array}{c}\text { Incomplete Description } \\
(n=75)\end{array}$ & $\begin{array}{c}\text { Complete Description } \\
(n=65)\end{array}$ & $t$ value & Significance \\
\hline Claritin-D & 4.65 & 4.00 & 2.64 & .009 \\
Cadura & 4.37 & 3.77 & 2.88 & .005 \\
MetroGel & 4.29 & 3.53 & 3.63 & .001 \\
Fosamax & 4.52 & 3.89 & 2.72 & .007 \\
Imitrex & 4.63 & 4.13 & 2.33 & .021 \\
Relafen & 4.66 & 3.80 & 3.61 & .000 \\
Sporanox & 4.25 & 3.80 & 1.97 & .050 \\
Atrovent & 4.51 & 4.17 & 1.60 & .113 \\
\hline
\end{tabular}

${ }^{a}$ Seven-point scale with endpoints of $1=$ "Extremely likely to recommend or purchase" to $7=$ "Extremely unlikely to recommend or purchase."

regardless of risk statement completeness, respondents indicated that they were more rather than less likely to purchase or recommend a described drug.

\section{Discussion}

Study One demonstrated that adoption of a 3\% base level for side effect reporting has a significant effect on a drug's appeal, as measured by consumers' rating of their likelihood to recommend or purchase the drug. This finding is intuitively reasonable. Drugs that have more side effects should be less appealing than drugs with a greater number of associated side effects. However, although the more complete risk statements resulted in relatively lower intent to recommend or purchase versus the incomplete descriptions, the absolute level of intent to recommend or purchase nevertheless was unexpected. It might be expected that consumers' ratings of drugs with fewer side effects (i.e., the incomplete risk statements) would fall above the neutral scale point on the "recommend or purchase" rating scale, whereas the ratings of drugs with more side effects (i.e., the complete risk statements) would fall below the neutral scale point. This was not the case.

The significant differences in consumers' reactions to complete and incomplete side effect descriptions (as evidenced in this study) lend support to the belief that completeness in side effect reporting does affect drug perceptions and preference. However, given the finding that all described drugs were likely to be recommended or purchased, an attempt was made to identify those factors that might have influenced consumers' positive responses. Identified explanations relate to consumers' mind-set and methodology.

One reviewer pointed out that consumers' mind-set with regard to advertised drugs may be one contributing factor to the pattern of response. Consumers may have reacted positively to all advertised drugs in this study because they believe that a drug must be "good" if it is allowed to be advertised. Thus, it would be appropriate to recommend or purchase any advertised drug; the only evaluation needed would be to identify the drug that may be the "best" among all acceptable choices. We believe that this is an intuitively plausible identification of one contributing 
factor, supported by recent survey data that indicate that about half of consumers believed that DTC ads must receive government approval prior to display and $43 \%$ of consumers believe that only "completely safe" drugs are allowed to be advertised (Bell, Kravitz, \& Wilkes, 1999).

Although consumers' mind-set may have played a role in the pattern of results, we believe that methodological issues also may have contributed. As a result, we focused our efforts on identifying methodological aspects of Study One that may have increased the likelihood of all drugs being recommended or purchased. Two aspects of the methodology were identified:

- First, each of the prescription drugs were presented in isolation; that is, there was no "head-to-head" comparison of the same drug when presented with a complete and an incomplete description. Without this comparison, respondents may have assumed that all advertised drugs were safe and therefore confidently could be "recommended or purchased."

- Second, all side effects were preceded by an imprecise frequency descriptor (Maat \& Klaasen, 1994; Toogood, 1980), that is, words such as "some," "many," and "few" rather than an absolute numeric descriptor. In the absence of numeric levels of incidence, consumers in Study One might have assumed that regardless of the number of side effects listed in the risk statement, the absolute likelihood of exhibiting any particular side effect was low. Beyond the ambiguity of imprecise frequency descriptors, consumers' assumption that all side effect levels were low may have been facilitated by some of the copy used in the risk statements, for example, "Though most users experience trouble-free relief," other, less frequently reported side effects are, "and while most people tolerate Relafen well. . .."

If consumers made either of the prior assumptions, then the results of Study One appear more understandable. However, to help resolve the issue of why all drugs received positive ratings regardless of risk statement completeness, Study Two explored the effects of risk statement completeness from a different methodological perspective.

\section{Methods: Study Two}

Study Two examined the effect of risk statement completeness with a methodology that addresses some of the potential underlying reasons for the overall positive perceptions of all described drugs in Study One. The changes in Study Two related to drug comparisons, minimal levels of side effects reported, and the presence of numeric information:

- Drug comparisons. Study Two presented paired comparisons of drugs. The same respondent was given a complete and incomplete risk statement for different drugs that provided the same health benefit. This "head-to-head" comparison allowed consumers to compare directly drugs with the same benefits but with different sets of associated risks.

- Minimal levels of side effect reported. As discussed earlier, advertisers have the latitude to choose the side effects reported in the risk statement. Study One set a minimal level of reporting (3\% incidence or higher). Study Two operationalized "completeness" from the advertiser perspective and permitted each advertiser to set the minimal level of reported side effects. A complete risk statement in this study was defined as "a statement that contains all side effects with an incidence greater 
TABLE 4 Product Descriptions and Risk Statements Used in Study Two

\section{Case 1}

Incomplete Description

Complete Description

Complete Description

Case 2

Incomplete Description

Complete Description

\section{Case 3}

Incomplete Description

Complete Description

\section{Case 4}

Incomplete Description

Complete Description
Atro is a prescription drug product that helps treat runny noses. The potential side effects associated with Atro are: mild nose bleeds $(9 \%)$ and nasal dryness $(5 \%)$.

Nevo is a prescription drug product that helps treat runny noses. The potential side effects associated with Nevo are: headache $(10 \%)$, upper respiratory tract infections $(10 \%)$, mild nose bleeds $(9 \%)$, sore throat $(8 \%)$, and nasal dryness $(5 \%)$.

Tarlex is a prescription drug product that helps with genital herpes. The potential side effects associated with Tarlex are: nausea (15\%), headache $(14 \%)$, diarrhea (5\%), and dizziness $(3 \%)$.

Reval is a prescription drug product that helps with genital herpes. The potential side effects associated with Reval are: nausea (15\%), headache $(14 \%)$, vomiting $(6 \%)$, diarrhea $(5 \%)$, constipation $(4 \%)$, abdominal pain (3\%), and dizziness (3\%).

Oliva is a prescription drug product for breast cancer therapy. The potential side effects associated with Oliva are: hot flashes $(48 \%)$, vaginal discharge $(30 \%)$, and irregular menses $(25 \%)$.

Nadex is a prescription drug product for breast cancer therapy. The potential side effects associated with Nadex are: hot flashes (48\%), weight gain (38\%), fluid retention $(32 \%)$, vaginal discharge $(30 \%)$, nausea $(26 \%)$, and irregular menses $(25 \%)$.

Prava is a prescription drug product that helps prevent a first heart attack. The potential side effects associated with Prava are: slight rash (4\%) and mild upset stomach $(3 \%)$.

Choval is a prescription drug product that helps prevent a first heart attack. The potential side effects associated with Choval are: localized musculoskeletal pain $(10 \%)$, common cold $(7 \%)$, nausea/vomiting $(7 \%)$, headache $(6 \%)$, diarrhea $(6 \%)$, abdominal pain $(5 \%)$, constipation $(4 \%)$, fatigue $(4 \%)$, general chest pain $(4 \%)$, cardiac chest pain $(4 \%)$, slight rash $(4 \%)$, rhinitis $(4 \%)$, dizziness $(3 \%)$, and mild upset stomach $(3 \%)$. 
TABLE 4 Continued

\section{Case 5}

Incomplete Description

Complete Description
Larit is a prescription drug product for children that helps relieve seasonal allergy symptoms. The potential side effects associated with Larit are: hyperactivity (3\%) and drowsiness $(2 \%)$. Claris is a prescription drug product for children that helps relieve seasonal allergy symptoms. The potential side effects associated with Claris are: nervousness $(4 \%)$, hyperactivity $(3 \%)$, wheezing $(4 \%)$, and drowsiness $(2 \%)$.

than or equal to the side effect with the lowest incidence mentioned in the advertiser created risk statement."

- Presence of numeric information. Numeric incidence levels accompanied all reported side effects.

Each of these aspects of Study Two are reflected in the experimental materials.

\section{Creation of Complete and Incomplete Risk Statements}

Two different risk statements were developed for each of five drugs advertised in DTC prescription drug ads. The first risk statement (for analytical purposes labeled the "incomplete risk statement") was taken directly from the drug's DTC advertising. In all cases, these descriptions presented a subset of all potential risks and side effects. A second risk statement for the drug (for analytical purposes labeled the "complete risk statement") presented a more complete description. Here, reflecting this study's operational definition of completeness, all side effects with an incidence level greater than or equal to the lowest incidence level in the advertiser-created description were included. Numeric descriptors accompanied all side effects in both risk statements. Both incomplete and complete risk statements were preceded by a brief description of the condition treated by the drug and the drug's primary benefit(s). Finally, because consumers were asked to make 'head-to-head' comparisons, a fictitious name was given to each of the described drugs. The risk statements for each of the five drugs used in this study are shown in Table 4.

\section{Questionnaire}

The five-page questionnaire contained five sets of paired product descriptions, one set presented on each page. In the development of the questionnaire, a coin toss determined the ordering within each pair of descriptions, that is, whether the complete or incomplete risk statement was presented first. The random ordering of descriptions within each pair was done to reduce presentation order bias, that is, having complete or incomplete descriptions appear in the same order across the entire questionnaire.

\section{Measures}

Two measures followed each set of paired product descriptions. The first measure assessed perceptions of product safety and read as follows: 
TABLE 5 Study Two: Safety Ratings as a Function of Side Effect Description Completeness

\begin{tabular}{cccrc}
\hline \multicolumn{4}{c}{ Mean Rating $^{a}$} & \\
Case & Incomplete Description $^{b}$ & Complete Description $^{b}$ & $t$ value & Significance \\
\hline 1 & 5.04 & 2.39 & 7.44 & .000 \\
2 & 5.47 & 3.36 & 5.57 & .000 \\
3 & 4.86 & 2.51 & 6.77 & .000 \\
4 & 7.03 & 2.31 & 10.62 & .000 \\
5 & 6.59 & 3.02 & 9.42 & .000 \\
\hline
\end{tabular}

${ }^{a} n=58$.

${ }^{b}$ Ten-point scale where $0=$ "Not at all safe" and 9 represents "extremely safe."

On a scale of 0 to 9 , where 0 means "not at all safe" and 9 means "extremely safe," how would you rate the safety of each drug?

I would give [insert name of drug from first description] a rating of

I would give [insert name of drug from second description] a rating of

The second measure, similar to Study One, asked each respondent to make a judgment related to drug recommendation or purchase. Respondents were asked to select (from among the two described drugs) the one that they would recommend or purchase, as follows:

If I were to purchase or recommend a drug for treating [insert name of medical condition], I would choose (place an $\mathrm{X}$ by the drug you would choose):

(Name of drug from first product description)

(Name of drug from first second description)

\section{Procedure}

Respondents completed each questionnaire individually. Recruited respondents were taken to a small interview room and were asked to read the survey directions and complete the survey. Respondents were given an unlimited amount of time to complete the survey, which was given to a trained interviewer upon completion. Surveys were checked for completeness. Two problem questionnaires (more than one item checked on the personal recommendation scale) were returned to the respondent for revision.

\section{Results}

Demographic Characteristics. The sample for Study Two consisted of 58 adults recruited via mall intercept. No incentive was offered for study participation. Similar to the sample of Study One, the sample for Study Two (Table 2) consisted of approximately equal numbers of men and women with a broad range of education, age, and income. 
TABLE 6 Study Two: "Intent to Recommend or Purchase" as a Function of Side Effect Description Completeness

\begin{tabular}{ccccc}
\hline & \multicolumn{2}{c}{ Percent of Respondents Selecting } & & \\
\cline { 2 - 3 } Case & Incomplete Description & Complete Description & $x^{2}$ & Significance \\
\hline 1 & $79.3 \%$ & $20.7 \%$ & 19.92 & .000 \\
2 & 82.8 & 17.2 & 24.96 & .000 \\
3 & 84.5 & 15.5 & 27.61 & .000 \\
4 & 77.6 & 22.4 & 17.67 & .000 \\
5 & 98.3 & 1.7 & 54.12 & .000 \\
\hline
\end{tabular}

${ }^{a} n=58$.

Effect of Risk Statement Completeness on Perceptions of Product Safety and Appeal. Table 5 presents the mean safety ratings for each of five drugs when the drug's description is accompanied by an incomplete and complete risk statement. Table 5 also presents the results of $t$ tests comparing these means. With regard to the absolute level of mean scores, level of completeness affected perceptions of drug safety. Mean scores for drugs described with the incomplete description always fell on the positive (i.e., "more safe") end of the rating scale, whereas the mean scores for drugs presented with the complete description fell on the neutral to negative (i.e., "less safe") end of the scale. Differences all were statistically significant ( $t$ tests were all significant at $p<.001$ ); drugs described with incomplete descriptions of side effects always were rated as more safe.

Given this pattern of response, it is not surprising that respondents significantly were more likely to recommend or purchase a drug described with an incomplete risk statement (Table 6). Across all five drug pairings, a minimum of three-quarters of the sample preferred the drug presented with an incomplete description of side effects. Chi-square tests indicate that levels of preference were, in all cases, significantly different than chance, that is, a preference of $50 \%$ (all $p<.001)$.

\title{
Discussion
}

DTC prescription drug advertising has polarized the medical community. Some argue that DTC advertising is "an excellent way to meet the growing demand for medical information, empowering consumers by educating them about health conditions and possible treatments" (Holmer, 1999). Similarly, an FDA/Prevention Magazine research study concluded that

\begin{abstract}
Many consumers who have seen advertisements for medicines they are currently taking say the advertising makes them feel better about the medicine they're taking, makes them more likely to take their medicine and reminds them to have their prescriptions refilled. [These outcomes] may play a very real role in enhancing public health. "National Survey," 1998)
\end{abstract}

Others argue that DTC drug advertising undermines the patient-physician relationship (Hollon, 1999), increases the likelihood of overmedication, increases consumers' preference for drug versus other therapies, increases consumers' demand for more 
expensive drug treatments versus other treatment options, and wastes the time of both patients and doctors (Brody, 1998; Freudenheim, 1998). Hoffman and Wilkes (1999) summarized this point of view when they stated that DTC advertisements "are intended not to educate patients, nor to empower them to be more intelligently involved in their own care, but merely to increase physician prescribing, regardless of need (p. 1301)."

We believe that DTC drug advertising has the potential to serve the public good. In addition to the reasons articulated earlier in support of DTC advertising, consumers need the information presented by DTC advertising because "managed care makes healthcare less personal and also imposes greater demands on patients to keep track of their own medical needs" (Pines, 1997, p. 65). But we believe also that the potential benefits of DTC advertising have not yet been realized because of the poor quality of information presented in the advertising. According to the FDA/ Prevention Magazine research study only $28 \%$ of the people who viewed DTC ads felt strongly that they were educated about the risks and benefits of prescription drugs, and a majority (55\%) felt the ads made the drugs appear harmless ("National Survey," 1998). In sum, because consumers need "more and better information to maintain good health" (Pines, 1997), the potential benefits of DTC drug advertising will be realized only when the information communicated by the advertisements provides a sound basis for helping consumers understand (a) the most appropriate circumstances for drug usage and (b) the benefits and risks associated with drug usage.

The FDA/Prevention Magazine finding that a majority of consumers believe that DTC advertising makes the advertised drugs appear harmless is directly related to the outcome of this research. ("National Survey," 1998). Studies One and Two explored the relationship between the completeness of the statement describing drug-associated side effects and consumers' perceptions of that drug's safety and appeal. Although the findings need to be tempered by two study limitations (relatively small sample sizes and the fact that not all respondents were in a drug's target audience), the research strongly suggests that there is a direct relationship between completeness in side effect reporting and consumers' perceptions of drug safety and appeal. Studies One and Two explored this relationship from different perspectives and methodological approaches, yet the fundamental finding of both studies was the same: Consumers rate the safety and appeal of drugs described with an incomplete disclosure of side effects significantly more positively than comparable drugs described with more complete side effect disclosure. It appears, therefore, that advertisers' presentation of incomplete risk statements do not allow consumers to evaluate adequately drug risks and benefits and, as a result, prevent the positive potential of DTC drug advertising from being realized.

It is important to note that the inaccurate conclusions many consumers draw from incomplete risk statements (with regard to drug safety and appeal) likely are to become even more problematic as DTC advertisers change the form and content of the brief summary that accompanies print DTC drug ads. In the past, the brief summary presented tables and text that fully described the range and levels of drugassociated side effects so that those (likely few) consumers who desired could find out the "full story." Brief summaries recently have changed to be more consumer friendly. New, revised brief summary content typically eliminates data tables and complete descriptions of drug-related side effects. As a result of these changes, all consumers are unable to determine whether the risk statement accurately communicates the range and breadth of drug-related side effects. 


\section{Implications for the Regulation of DTC Prescription Drug Advertising}

We believe that the research findings, consistent across both studies, provide clear support for a reexamination of what constitutes "fair balance" in DTC prescription drug advertising. Fair balance is, and should be, an important component of DTC prescription drug advertising. Fair balance is required because the FDA seeks to maximize the chances that consumers are able to make a fair, informed, and reasonable evaluation of an advertised drug's safety and appeal, and this type of evaluation can occur only if consumers have a complete understanding of the advertised drug's benefits and risks. This research suggests that the latitude currently accorded DTC drug advertisers prevents the goal of fair balance (and informed evaluations) from consistently being achieved. As demonstrated in the research, it is entirely possible for consumers to draw false conclusions from DTC drug advertising, for example:

- Two equivalent drugs (with equivalent benefits and risks) may be perceived differentially by consumers because of differences in the number of side effects presented in their risk statements.

- Drugs may appear to be safer then they actually are because of the number and types of side effects included in their risk statement.

The research strongly suggests that consumers might well be at risk given the FDA's current approach to the evaluation of fair balance; consumers are making decisions and requesting drugs from their physicians based on incomplete information and false perceptions of relative drug safety.

The trend at the FDA is for a loosening of regulations governing DTC prescription drug advertising, as evidenced in the 1997 revision of guidelines for television advertising. The research findings suggest that this is the wrong direction to take at this time. Consumers appear determined to take a more active role in their health care decisions. For these decisions to be informed, consumers need accurate, meaningful, and complete information. We agree with Hollon (1999), who states, "Providing poor quality information in today's marketplace of health information results in little or no benefit for the public.” (p. 383)

\section{Implications for Advertiser Practice}

Until the FDA revises its "fair balance" regulations, consumers must rely upon advertiser self-regulation for improvements in the content and communication of DTC drug advertisements. The argument can be made that social and ethical considerations require such self-regulation.

Incomplete risk statements can be considered unethical advertiser behaviors if they are found to violate any of the well-accepted moral (as opposed to legal) criteria commonly applied to business (in general) and advertising (in particular). Here are two relevant principles (Feiser, 1996) and their application to DTC prescription drug advertising:

- Harm principle: Business should avoid causing unwanted harm. In the context of DTC prescription drug advertising, this principle applies to both an advertiser's business competitors and to consumers. Imagine two drugs that work identically and that have the same set of side effects. One drug is advertised with an incomplete risk statement and the second is advertised with a complete risk statement. Harm (through loss of sales) is done to the second drug's advertiser if consumers prefer the first drug over the second because of the difference in the number and 
types of side effects reported in each drug's risk statement. Psychological and physical harm also can be done to consumers should they request the first advertised drug over the second thinking that they can avoid some of the side effects associated with the second drug but then experience some of side effects not noted in the first drug's incomplete risk statement.

- Autonomy principle: Businesses should not infringe on the rational, reflective choices of people. Business should respect the right of each person to make a responsible choice. Incomplete risk statements would be shown to violate this moral principle if consumers make different brand decisions based on risk statement completeness. These decisions would neither be rational nor responsible because the decisions were made on the basis of incomplete information. Violations of this principle would be important especially as research has shown that consumers are more likely to make unsound medical decisions when they have incomplete or imperfect knowledge of the risks and benefits associated with alternative options (Viscusi, Magat, \& Huber, 1986). Importantly, although some might believe that an appeal to advertiser's ethics likely is to be unproductive, it is encouraging to note that advertisers in the highest levels of advertising agency management appear willing to incorporate ethical considerations in their advertising decision-making process (Davis, 1994).

\section{Limitations of the Research and Implications for Further Research}

The research findings provide consistent initial evidence of how the FDA's fair balance criterion can fail to protect consumers from incomplete risk statements. However, the limitations of the current research, coupled with the issues raised by the research, necessitate further research in the area.

One set of further research activities should address methodological issues. First, the relatively small, nonrandom sample in this research limits the strength of conclusions and lowers generalizability to the broader population. Further research will not only want to explore issues raised with more generalizable samples but additionally will want to select samples that permit reliable analyses of important subpopulations, for example, individuals in different demographic groups, individuals with varying experience with DTC prescription drug advertising, and individuals with varying experience with both advertised and nonadvertised prescription drugs. Second, the current research used a nonrandom sample to set a minimal level of consumer concern with drug-associated side effects. Although the research showed that this level did differentially affect consumers' responses to risk statements, further research is needed that will identify more systematically minimal levels of concern. Such research could determine, for example, if there is a minimal level of concern across all conditions treated by DTC prescription drugs or if a minimal level of concern varies across conditions.

Beyond methodology, a second set of research is needed to improve our understanding of the process by which consumers perceptions and attitudes are affected by DTC advertising and risk statements. Further research is needed to help better define "risk statement completeness." The two studies presented in this paper highlight the complexity of this issue, for example:

- Should "completeness" be defined in terms of the number of side effects reported? In terms of a minimum incidence level? In terms of the most common side effects? (And how is "most common" defined?) 
- Does "completeness" require numeric indicators in the risk statement? For example, is the statement, "The major side effects are headache and nausea" equivalent in terms of completeness with the statement, "The major side effects are headache $(66 \%)$ and nausea $(45 \%)$ ?"

We encourage research that explores the issues of "completeness" and "fair balance" from the consumers', rather than simply a content analysis, perspective. In addition, the current research used single measures ("recommend or purchase," "safety") to evaluate consumers' drug perceptions after exposure to complete and incomplete risk statements. Although there were methodological advantages to using single summary measures, one drawback of these measures was that they did not permit an in-depth exploration of why responses to these measures varied as a result of risk statement completeness. Therefore, further research is required that will assess reactions to risk statements that measure specific dimensions of "safety" and that probe the range of reasons why a particular drug is or is not "purchased or recommended."

\section{Conclusion}

There is little doubt that DTC prescription drug advertising will continue to increase. We believe that DTC prescription drug advertising is only in the consumer interest if it is constructed and presented in a way that helps consumers fully understand an advertised drug's benefits and side effects. As a result, from a regulatory perspective, we recommend strongly a reexamination of the FDA's current approach to "fair balance" and a redefinition of "fair balance" based on empirical research findings. Until this is done, we agree with Smith (1999), who argues that

"Pharmaceutical companies must now move beyond simply meeting FDA's fair balance requirements and seek the trust of consumers reading their ads. Copywriters need to select side effects that are most important for consumers to know about and put these risks into proper perspective." (p. 519)

\section{References}

Alperstein, N., \& Peyrot, M. (1993). Consumer awareness of prescription drug advertising. Journal of Advertising Research, 33(4), 50-56.

Bell, R. A., Kravitz, R. L., \& Wilkes, M. S. (1999). Direct-to-consumer prescription drug advertising and the public. Journal of General Internal Medicine 14, 651-657.

Brody, J. E. (1998, December 20). Consumers should approach ads for prescription drugs with caution. Houston Chronicle, p. 10.

Code of Federal Regulations. (2000). Prescription drug advertising. 21 CFR Part 202, 72-81.

CDER. (1999). Attitudes and behaviors associated with direct-to-consumer (DTC) promotion of prescription drugs: Main survey results. Available: http://www.fda.gov/cder/ddmac/ dtcindex.htm

Cohen, E. P. (1990). Are pharmaceutical ads good medicine? Business and Society Review, 2(Spring), 8-10.

Davis, J. J. (1994). Ethics in advertising decision-making: Implications for reducing the incidence of deceptive advertising, Journal of Consumer Affairs, 28(2), 380-402.

Everett, S. E. (1991). Lay audience response to prescription drug advertising. Journal of Advertising Research, 31(3), 43-49. 
Federal Trade Commission (FTC). (1983). Policy statement on deception. 45 A77R, October $27,689690$.

Feiser, J. (1996). Do businesses have moral obligations beyond what the law requires? Journal of Business Ethics, 15, 457-468.

Food and Drug Administration (FDA). (1999). Guidance for industry consumer: Directed broadcast advertisements. Available: http://www.fda.gov/cber/gdlns/advrts.pdf

Freudenheim, M. (1998, December 2). Prescription drug sales soar with help of advertisements. Oklahoma City Journal Record, p. 1.

Hoffman, J. R., \& Wilkes, M. S. (1999). Direct to consumer advertising of prescription drugs: An idea whose time should not come. British Medical Journal, 318(7194), 1301-1302.

Hollon, M. (1999). Direct-to-consumer marketing of prescription drugs: Creating consumer demand. Journal of the American Medical Association, 281(4), 382-384.

Holmer, A. F. (1999). Direct to consumer prescription drug advertising builds bridges between patients and physicians. Journal of the American Medical Association, 281(4), 380-382.

Liebman, M. (2000). Three scenarios for direct-to-success advertising. Medical Marketing and Media, 35(4), 72-76.

Maat, H. P., \& Klaasen, R. (1994). Side effects of side effect information in drug information leaflets. Journal of Technical Writing and Communication, 24(4), 389-404.

National survey of consumer reactions to direct-to-consumer advertising. (1998). Prevention Magazine.

Neergaard, L. (1999, February 3). Experts debate effect, of rise in prescription drug ads. Oklahoma City Journal Record, p. 1.

Okie, S. (2000, May 22). With TV spots, drug firms aim at patients' role: Strategy for prescriptions shifts away from doctors. The Washington Post, p. A1.

Perri, A., \& Nelson, A. (1987). An exploratory analysis: Consumer recognition of direct-toconsumer advertising of prescription medicines. Journal of Health Care Marketing, 7(March), 9-17.

Perri, M., \& Dickson (1988). Consumer reaction to a direct-to-consumer prescription drug advertising campaign. Journal of Health Care Marketing, 8(June), 66-69.

Peyrot, M., Alperstein, N. M., Van Doren, D., \& Poli, L. G. (1998). Direct to consumer ads can influence behavior. Marketing Health Services, 18, 27.

Pierpaoli, P. G. (1986). ASHPs position on direct-to-consumer advertising of prescription drugs. American Journal of Hospital Pharmacy, 43, 1763-1766.

Pines, W. L. (1997). New challenges for medical product promotion and its regulation. Food and Drug Law Journal, 52, 61-66.

Sheffet, M. J., \& Reece, B. B. (1994). Direct to consumer advertising of prescription drugs: Issues and concerns. In D. J. Ringold (Ed.), Marketing and public policy conference proceedings (pp. 5-9). Chicago: American Marketing Association.

Smith, D. (1999). Consumer trust takes more than fair balance. Pharmaceutical Executive, 19(4), S18-S19.

Toogood, J. (1980). What do we mean by “usually"? Lancet, 1, 1094-1102.

Viscusi, W. K., Magat, W. M., \& Huber, J. (1986). Informational regulation of consumer health risks: An empirical evaluation of hazard warnings. Rand Journal of Economics, 17, 351-365.

Zendle, L. (1999, November 26). TV ads for prescription drugs raising consumer interest. Salt Lake City Tribune, p. A13. 